\title{
Hawley or vacuum-formed retainers following orthodontic treatment?
}

\author{
Abstracted from \\ Mai W, He J, Meng $H$, et al. \\ Comparison of vacuum-formed and Hawley retainers: a systematic review. \\ Am J Orthod Dentofacial Orthop 2014; 145: 720-727. \\ Address for correspondence: Na Kang, Department of Orthodontics, College of Stomatology, \\ Guangxi Medical University, Nanning, Guangxi, China 530021. E-mail: kangna78@gmail.com
}

\section{Question: Are vacuum-formed retainers (VFRs) more effective than Hawley retainers (HRs) following orthodontic treatment?}

Data sources Cochrane Central Register of Controlled Trials (CENTRAL), Medline, Embase, ISI Web of Science, LILACS. In addition, Pro-Quest Dissertation and Thesis database and Pro-Quest Science Journals. Hand searches were also carried out in American Journal of Orthodontics and Dentofacial Orthopedics, Angle Orthodontist, European Journal of Orthodontics and Journal of Orthodontics. Study selection Two reviewers independently selected studies, and randomised, quasi-randomised (RCTs) and controlled clinical trials (CCTs) were considered. Studies with at least six months follow-up were included.

Data extraction and synthesis Data extraction and risk of bias assessment were carried out independently by two reviewers. A narrative summary was presented as a meta-analysis could not be performed.

Results Seven studies were included (five RCTs, two CCTs). Three were considered to be at low risk of bias, three at moderate risk and one at high risk. There was some evidence to suggest that no difference exists to distinguish between the HRs and VFRs with respect to changes in intercanine and intermolar widths after orthodontic retention. There was insufficient evidence to support the use of VFRs over HRs in relation to occlusal contacts, cost effectiveness, patient satisfaction and survival time.

Conclusions This systematic review suggests that further high-quality RCTs regarding the differences between HRs and VFRs during orthodontic retention are necessary to determine which retainer is the better selection for orthodontists.

\section{Commentary}

The familiar lament at the conclusion of any systematic review is the need for further high quality evidence. The truth is that the process of review involves the collation of published literature to date and the fact that the included studies, whenever they were published, would have constituted the best possible evidence at that given point in time. It would be utopian to have structured research and well randomised studies on any subject, but yet the variation of published literature tends to add an element of diversity, and the authors have a challenge before them in arriving to conclusions even while recommending the need for more quality evidence. From that perspective the present review is contemporary and would find an echo in the thinking of most clinicians.

Retention is now construed as a critical phase of orthodontic treatment because of the tendency of teeth to return to untreated positions for a host of factors from gingival, periodontal influences to continued growth. While the need for retention has been accepted universally, the methods and periods of retention are diverse and this is what clearly emerges from the current review. The social and aesthetic influence has a significant effect on the choice of retainers and their usage. The introduction of the Hawley retainer in 1919 led to its widespread acceptance and it remains popular today. The compelling simplicity and invisibility of the vacuum-formed retainer introduced in 1971 has led to an increasing popularity. The economic issues would also add to the growing popularity of the VFR (Vacuum Formed retainer). Again a critical appraisal of available literature reveals that to date there is no compelling evidence to support the VFR over the HR (Hawley Retainer). With this backdrop the value of the SR and the efforts of the authors need to be lauded in trying to provide some leading directions in a common clinical intervention.

The authors have laid fairly rigorous inclusion criteria with only randomised or quasi-randomised clinical trials being included in the review. Patients who had maxillary/mandibular or both retainers were included in the study, with no reference to the type of active orthodontic treatment, full coverage from molars to molars and a sixmonth follow-up in retention. Only VFRs or HRs were included and the primary outcomes studied were the Little Index, the intermolar and intercanine archwidths and arch length. The authors have expanded the envelope by looking at secondary outcomes of cost effectiveness, occlusal contacts, patient satisfaction and the impact on gingival and periodontal health. Any questions that could have been raised by the discerning clinician have been addressed. The search criteria and databases covered are comprehensive.

The processes defined in the study are stringent with two reviewers independently reviewing the material and a process of resolution of conflict with a $3^{\text {rd }}$ review author on the team. It is interesting to note that by addressing six domains, the studies have been classified into low, moderate and high risk of bias. The statistical methods have a detailed justification by the authors as to why a meta-analysis is not possible and again this comes as no surprise because of the diversity of studies and the parameters studied in each of them.

From an initial 89 studies that could have been relevant to the review, only seven studies met the inclusion criteria and are included in the review. All the studies are parallel group studies and 
five are randomised clinical trials, two are clinical controlled trials. Four studies have evaluated the specified outcomes.

From the classic perspective there is large dissonance in the interventions in the selected studies and comparisons would be difficult to derive. The wearing of vacuum formed retainers varies from 24 hours a day for the first week and then 12 hours a day for the rest of the study period. The variance is clearly discernable in both the frequency of wear and the duration of both VFRs and HRs with the duration also varying from six to 12 months. In one study the use of HRs has been combined with mandibular lingual bonded retainers. It is not surprising that a statistically significant result has been seen in terms of patient satisfaction and embarrassment with HRs in one study. A comparison of the VFR group, the HR group and fixed appliances also has a predictable sway of balance towards the VFRs. The effectiveness of HRs and VFRs comes from three studies with a low to moderate risk of bias. One study derives that VFRs are more effective at retaining the maxillary and mandibular anterior segments while the others seem to state that both methods of retention are equally effective. The occlusal contacts are more with the HRs and predictable so as the HR will permit a settling of the posterior segments. The complete occlusal coverage with the VFR would not permit the posterior segment to settle vertically but the continued mobility of teeth on removal of VFRs permits a settling and over an extended period of time the outcomes converge. The other issue that unveils itself is that if VFRs are the retainers of choice then what thickness and what type of material would be better. There is large variability of the thickness of materials used from $0.75,1.0$ and $1.5 \mathrm{~mm}$, and the type of thermoplastic material used would have an impact on the wear resistance and durability. Studies have shown that hard polyethylene terephthalate glycol polymers show greater wear resistance than the softer polypropylene based thermoplastics.

The heterogeneity of the included studies has led to a narrative review. The inconsistency of measured outcomes and standard periods of wear, comparisons all lead to the conclusion that a metaanalysis is not possible. The review highlights several issues; there is a lack of consensus in the oldest specialty regarding the method, duration of wear and the period of retention. Both methods of retention, that is VFRs and HRs, are effective, that while the evidence is sparse and lacking it may be possible to opine that aesthetics, compliance and patient acceptability along with cost effectiveness would tip the balance in favour of VFR and this is the increasingly popular retention appliance today.

The present systematic review brings into sharp focus the debate on randomised controlled trials and the humble retrospective study. It is easily accepted that retrospective investigations would have a high risk of bias, inconsistency in design and heterogeneity of data that eventually emerges from them, yet the information that unveils itself is important and clinically relevant. This comes forth from the present review, while the authors tread cautiously in presenting an outcome, the inferences are clearly there for the clinician.

The biggest problem with the classic retrospective study is the availability of records, as the study would be completely reliant on this. The design may reflect the thinking and issues at a certain point in time and as GB Shaw reflects that for every problem science solves, it creates ten others. The prospective study gives the opportunity to plan, to measure, to create quantifiable data that may be merged into a meta-analysis. It is the ideal answer for answering crucial issues, but the history of humanity has been written from sifting of artifacts and relics and it should be no different in orthodontics. The review is a great effort and needs to be both read and ruminated upon. The fairly familiar litany of insufficient quality evidence at the end of the review needs to be interpreted in the light of the facts presented. It is evident that future studies should address the type of VFRs, the period of wear, and comparisons to other established forms of retention such as the lingual bonded retainers.

As of now:

1. Vacuum formed retainers and Hawley retainers are equally effective in retention following fixed orthodontic therapy.

2. If aesthetics, patient sensitivity, compliance and economics be the benchmarks then the scales begin to tip in favour of the VFR.

Retention continues to be a mystery and an eternal controversy in orthodontics. The answer to the enigma of retention lies in more structured and focused research.

\section{Anmol S. Kalha ITS Dental College, Hospital and Research Center, Greater Noida, NCR, India}

Evidence-Based Dentistry (2014) 15, 110-111. doi:10.1038/sj.ebd.6401061 